\title{
ELECTROCARDIOGRAMS IN TRAUMATIC SHOCK IN MAN
}

\author{
By CHARLES H. BURNETT, EDWARD F. BLAND, AND HENRY K. BEECHER
}

(From the Mediterranean Theater of Operations, U. S. Army)

(Received for publication February 27, 1945)

As traumatic shock develops, cardiac output falls. There are several fairly well-established explanations for this. It is usually stated, however, that this diminishing output is due to phenomena arising outside of the heart and that the heart itself remains unimpaired until very late. We had hoped to obtain evidence that would, insofar as could be judged by electrocardiograms, give information clearly for or against this view. It was thought likely that as a result of the inadequate circulating blood volume, the lowered blood pressure, and the compensatory tachycardia, the coronary circulation might suffer to an extent recognizable by electrocardiogram.

We are recording herewith observations on a series of 58 electrocardiograms obtained on 30 seriously-wounded soldiers in traumatic shock. The tracings were assembled at the 94th Evacuation Hospital in February and March of 1944, during the operations on the Cassino Front, and subsequently in April and May, on the Anzio Beachhead. When possible, tracings were made both during shock and in the recovery phase. In 10 patients (one-third of the group), the blood pressure could not be measured on entry. In the remaining two-thirds the degree of circulatory collapse was somewhat less severe as reflected by blood pressures of 60 to $70 \mathrm{~mm}$. $\mathrm{Hg}$ systolic and 20 to $40 \mathrm{~mm}$. Hg diastolic. Blood pressure determinations were made with a mercury manometer or with aneroid manometers checked against the mercury.

\section{THE ELECTROCARDIOGRAMS}

Definite abnormalities of the electrocardiograms were encountered in 5 of the 30 patients studied, but the most striking feature was the normal character of most of the tracings (in 25 of the 30 patients). These normal records were observed in many of those in extreme shock as well as in those

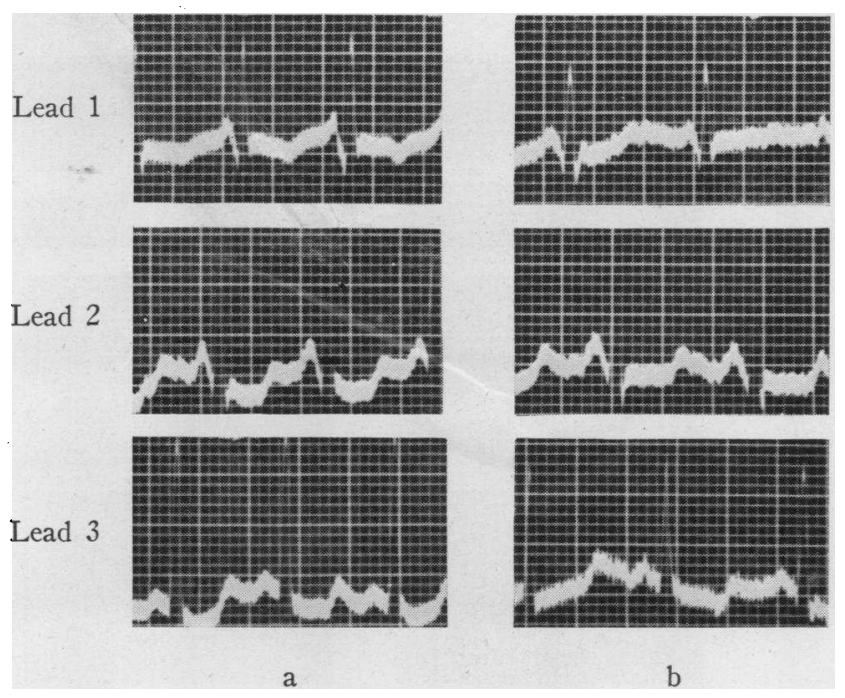

Fig. 1. Electrocardiograms-Case 1

a. Showing late inversion of the $\mathrm{T}$ waves in lead 1 , and diphasic $\mathrm{T}$ waves in leads 2 and 3.

b. From the same patient taken 30 minutes later after the shock had been relieved. It shows a striking improvement in the $T$ waves, especially in lead 1 . 
in better condition. However, in the 5 patients where abnormalities were recorded, they are of sufficient interest to warrant a brief discussion.

Abnormal $T$ waves. A definite but transient inversion of the $\mathrm{T}$ wave in lead 1 was observed in 2 instances.

Case 1. J. B. C., age 27 , received a severe wound of the left chest with open pneumothorax. He was brought to the hospital within a few hours after emergency treatment in the field. On arrival he was in profound shock with unmeasurable blood pressure (hemoglobin 13 grams, hematocrit 38). The electrocardiogram taken at this time showed a sharp late inversion of $\mathrm{T} 1$ and moderate depression of the S-T interval in leads 2 and 3 (Figure 1a). Following administration of about a liter of whole blood in a half hour, his circulatory state improved promptly and his blood pressure had risen from 0 to $125 \mathrm{~mm}$. $\mathrm{Hg}$ systolic and $65 \mathrm{~mm}$. Hg diastolic. A second electrocardiogram (Figure 1b) taken 30 minutes after the first showed a remarkable improvement in $\mathrm{T} 1$ to a low but upright position, and a lessening in the diphasic contour of S-T 2 and 3. There was no evidence of cardiac or pericardial injury in this case, and the prompt return of the inverted $\mathrm{T} 1$ towards normal coincident with improvement in the general circulation suggests that mechanical trauma of the heart was not a factor in this temporary abnormality. Subsequent to operation the clinical course insofar as known was uneventful.

Case 2. A. H., age 45 , received a penetrating wound of the left chest. Upon arrival at the hospital a few hours later, he was in severe shock. His blood pressure was $55 \mathrm{~mm}$. $\mathrm{Hg}$ systolic and the diastolic level was undetectable (hemoglobin 11.5 grams, hematocrit 34). An electrocardiogram (Figure 2a) revealed a sharp late inversion of the $T$ waves in lead 1 without other abnormality. A superimposed artefact due to alternating current is well displayed in this and the subsequent tracing. It was a frequent complication in the tracings taken on the Anzio Beachhead and at times difficult to eliminate. Following administration of 1 unit of albumin and 500 ml. whole blood, the patient responded satisfactorily. At the end of an hour, his blood pressure had risen to $90 \mathrm{~mm}$. $\mathrm{Hg}$ systolic and $60 \mathrm{~mm}$. $\mathrm{Hg}$ diastolic. An electrocardiogram (Figure 2b) showed a return to normal of the previously inverted $T 1$. There was again no evidence of lasting cardiac trauma and the subsequent course was uneventful.

Abnormal axis deviation (temporary). In planning this study we had anticipated encountering electrocardiographic evidence of temporary strain upon the right ventricle (right axis deviation) as a result of liberal use of intravenous fluids (plasma and blood) in combatting peripheral circulatory collapse. The following case is the only instance in which right axis deviation was noted.

Case 3. H. G. K., age (c.) 25, received a penetrating wound of the right chest with pneumothorax and retained foreign body. Upon arrival at the hospital he was in moderate circulatory collapse (hemoglobin 11.2 grams, hematocrit 33). His blood pressure was $65 \mathrm{~mm}$. $\mathrm{Hg}$

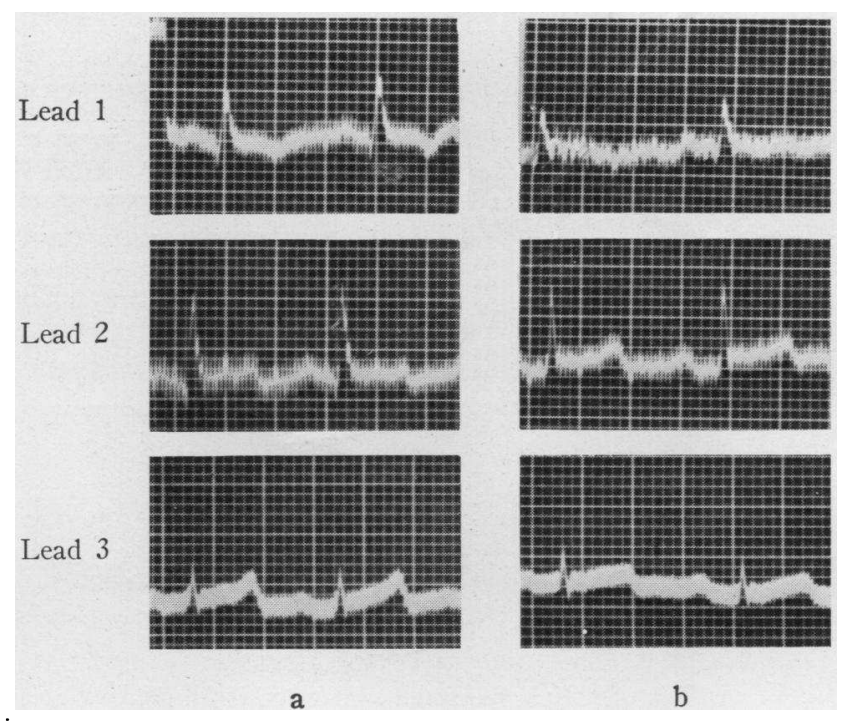

Fig. 2. Electrocardiograms-Case 2

a. In traumatic shock, showing late inversion of the $T$ waves in lead 1.

b. From the same patient 1 hour later showing improvement in the $\mathrm{T}$ waves coincident with relief of circulatory collapse. 


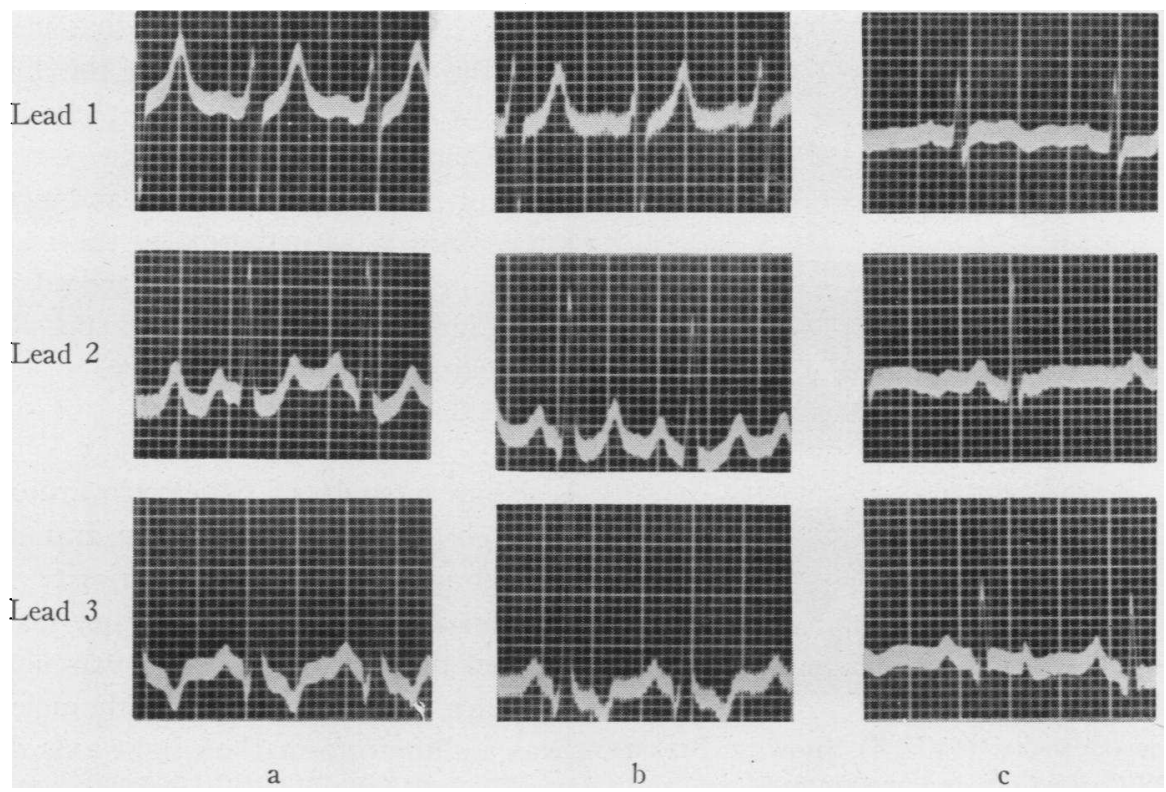

Fig. 3. Electrocardiograms-Case 3

a. Showing well marked right axis deviation.

b. After 30 minutes showing no change.

c. Following operation and 24 hours after the first tracing. It now shows normal axis deviation.

systolic and $20 \mathrm{~mm}$. $\mathrm{Hg}$ diastolic, and an electrocardiogram (Figure 3a) showed well-marked right axis deviation. There was no evidence in the liver or in the peripheral veins of right-sided heart failure. At the end of 30 minutes his circulatory state had deteriorated, in that his systolic blood pressure had dropped to $40 \mathrm{~mm}$. $\mathrm{Hg}$ and the diastolic level was unobtainable. An electrocardiogram at this time showed no change (Figure $3 \mathrm{~b}$ ). He received $1000 \mathrm{ml}$. whole blood and $500 \mathrm{ml}$. of 2 per cent sodium bicarbonate solution intravenously in $2 \frac{1}{2}$ hours preceding operation without signs of heart failure. The chest wound was successfully closed, and 18 hours after operation (and 24 hours after the original tracing) his condition was good. The blood pressure had returned to $110 \mathrm{~mm} . \mathrm{Hg}$ systolic and $70 \mathrm{~mm}$. $\mathrm{Hg}$ diastolic. The electrocardiogram showed a striking shift to normal of the axis deviation and concurrent changes in the contour of the $\mathrm{T}$ waves, the latter probably of no special significance. In the absence of other evidence of cardiac injury or strain, and the normal response of the heart to the intravenous infusion, we are inclined to interpret the changing electrocardiographic pattern in this patient as most likely of mechanical origin related to displacement (torsion) of the heart by blood, air, or injured lung prior to operation.

Miscellaneous abnormalities, including abnormal QRS complexes and cardiac irritability.

Case 4. L. K. R., age (c.) 25, received a penetrating wound of the left chest, perforation and hematoma of the left lung (lower lobe), laceration of the diaphragm and spleen, and multiple injuries of the extremities. Splenectomy was necessary shortly after arrival in the hospital. Post-operatively his condition was poor; in spite of supportive therapy, his blood pressure was $80 \mathrm{~mm}$. Hg systolic and $40 \mathrm{~mm}$. Hg diastolic. An electrocardiogram taken at this time ( 2 hours before death) (Figure 4$)$ showed abnormal W-shaped QRS complexes of low voltage, the explanation for which is not clear, but is probably related to extensive trauma in close proximity to the heart, possibly of the heart or pericardium.

Case 5. J. R. P., age 20, was wounded severely in the buttocks, genitals, and extremities. He was in extreme shock with unmeasurable blood pressure (hemoglobin 8.5 grams, hematocrit 25). Four hours after he was wounded, and 2 hours after he was hospitalized, he developed marked cardiac irritability, manifested by paroxysmal auricular fibrillation with a ventricular rate of 180 with short runs of ventricular tachycardia superimposed. Despite $500 \mathrm{ml}$. of plasma, $1750 \mathrm{ml}$. of whole blood and $500 \mathrm{ml}$. of 2 per cent sodium bicarbonate solution intravenously, his blood pressure had risen only to $78 \mathrm{~mm}$. $\mathrm{Hg}$ systolic and $50 \mathrm{~mm}$. $\mathrm{Hg}$ diastolic after 3 hours, and surgery was undertaken, the auricular fibrillation persisting. Following operation, he made a satisfactory recovery.

\section{DISCUSSION}

The abnormalities in the electrocardiograms recorded above are of some interest, and difficult to explain. In no instance did we observe clinical 


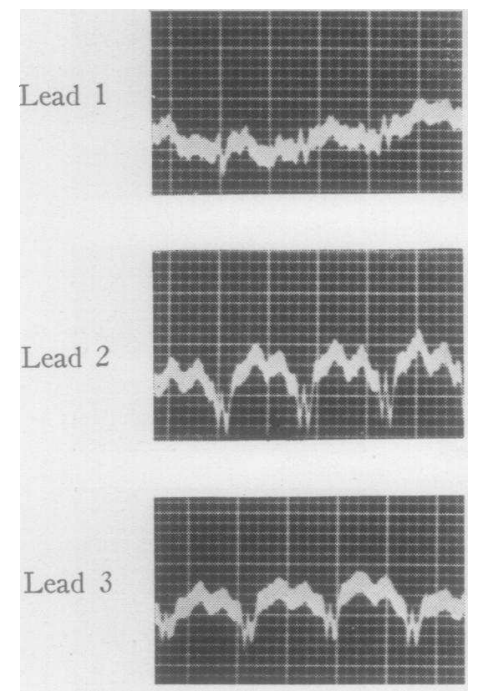

Fig. 4. Electrocardiogram (Case 4) Showing Bizarre QRS Complexes of Low Voltage

signs of cardiac weakness in the form of abnormal accentuation of the pulmonary second sound, basal râles, gallop rhythm, or congestion of the cervical veins or of the liver. As remarked above, the majority of the electrocardiograms were normal in character. Several patients in the series were in severe shock with low blood pressures for a period of hours with no effect upon the electrocardio- gram. It may be significant that in both patients with the transient inversion of the $T$ waves in lead 1 the wound involved the left chest, although as far as could be determined by x-ray and at the time of operation, the heart and pericardium escaped injury. Furthermore, the transient nature of the inversion was more in accord with a temporary functional disturbance (possibly hypoxia) than with lasting tissue injury.

\section{SU M M ARY}

From a study of 58 electrocardiograms on 30 severely wounded soldiers in traumatic shock it was found that: (1) the majority were normal; (2) in 2 instances striking, but transient, inversion of the $\mathrm{T}$ wave in lead 1 was noted; (3) in 1 instance, complicated by intrathoracic injury, there was a shift from marked right axis deviation back to normal following operation; (4) another patient showed bizarre QRS complexes of low voltage, and a fifth patient showed an unusual degree of temporary cardiac irritability with paroxysmal fibrillation and ventricular tachycardia. The possible significance of these changes has been discussed.

We are indebted to The Surgeon, Fifth Army, and to the Commanding Officer, 94th Evacuation Hospital, for their authorization of and co-operation in this study. 